\title{
An Immunological Hypothesis of Fu's Subcutaneous Needling Acupuncture
}

\author{
Wenbo $\mathrm{Xu}^{*}$, Jidong $\mathrm{Wu}$, Ping $\mathrm{Xu}$ \\ FSNAE, British Acupuncture Federation, Cambridge, United Kingdom
}

Received December 27, 2020

Revised March 28, 2021

Accepted April 6, 2021

Correspondence to

Wenbo Xu

FSNAE, British Acupuncture

Federation, Cambridge, United

Kingdom

E-mail wenbo-xu@hotmail.com
Fu's subcutaneous needling (FSN) is a novel acupuncture approach. Although it has been popularized and widely used by acupuncture practitioners, theoretical research on its mechanism of action remains lacking. This study establishes an immunological hypothesis for the efficacy of FSN basis existing knowledge in biomedical science, histology and physiology, and traditional acupuncture mechanisms. The theoretical inference indicates that FSN treatment may affect the body's immune system through subcutaneous connective tissue, similar to triggering a cobweb-like system. This article also proposes possible clinical implications of this theory, which require further studies to validate.

Keywords: Fu's subcutaneous needling, Acupuncture, Immune system, Connective tissue, Hypothesis, Cobweb

\section{INTRODUCTION}

Fu's subcutaneous needling (FSN) is a novel acupuncture therapy that involves inserting a modified acupuncture needle to stimulate subcutaneous loose connective tissue around "tightened muscles" or myofascial trigger points (MTrPs) to treat musculoskeletal complaints and other conditions [1]. FSN was invented by Dr Zhonghua Fu in 1996. FSN acupuncture has recently attracted several acupuncture practitioners owing to its therapeutic efficacy.

Currently, an isolated tightened muscle is the primary indicator for FSN acupuncture. Tightened muscles are identified as those that are tight and rigid on palpation when even fully relaxed. FSN acupuncture is based on the rationale that musculoskeletal and some other disorders are caused by tightened muscles, whereas tightened muscles are closely associated with the generation of MTrPs. An MTrP is a hyperirritable point in skeletal muscle that is associated with a hypersensitive palpable nodule [2]. FSN intervention relaxes the tightened muscles, which restores blood supply and consequently, relieves pain and resolves disorders.

The modified FSN needle is a trocar needle, which is a steel needle with a beveled tip and a plastic canula covering it. With a diameter of $1.05 \mathrm{~mm}$, this needle is 3 - to 5-times thicker than common acupuncture needles. Unlike traditional acupuncture techniques that involve perpendicular needle penetration through the skin, subcutaneous layer, and muscles and even reaching the bone, the FSN needle is horizontally inserted into the subcutaneous layer [3]. After needle insertion, needle manipulation on the subcutaneous layer is performed. The site of needle insertion is determined by the location of the tightened muscle. However, the insertion point is proximal but not in the affected area. FSN acupuncture does not entail a needling sensation (De-Qi).

Clinically, FSN acupuncture has been successful in treating musculoskeletal disorders, such as muscle or ligament injury or strains and often offers immediate pain relief. However, few clinical phenomena might be difficult to explain using the tightened muscle theory. For example, at times treatment yields symptom resolution or minimization but not muscle relaxation. Of interest, resolution of some internal organ disorders such as dysmenorrhea and ulcerative colitis has been observed with targeted treatment of tightened muscles. These phenomena are intriguing and require investigating the physiological effects of strong stimulation of subcutaneous tissues with a needle. The exact mechanism of action of FSN needs elucidation.

In 2017, we hypothesized that FSN acupuncture may exert its therapeutic effects by activating the immune system and tissue repair mechanism by stimulating the subcutaneous loose connective tissue [4]. This study further discusses, supplements, demonstrates, and details how the 'cobweb mechanism' could support this hypothesis. Furthermore, this article describes the theoretical basis and discusses the 
clinical significance of FSN acupuncture.

\section{IMMUNOLOGICAL BASIS OF FSN ACUPUNCTURE: THE COBWEB MECHANISM}

We propose the following mechanism of action of FSN acupuncture basis the current knowledge of biomedicine, physiology, histology, and immunology. FSN acupuncture mainly focuses on the subcutaneous layer, which is mainly composed of loose connective tissue. By stimulating the subcutaneous tissue, FSN acupuncture is considered to activate the immune system, which entails tissue inflammatory and anti-inflammatory activities, regeneration, and repair. These immunological activities involve removal of tissue necrosis, abnormal cells, and pathological factors that cause muscle tightening or ailments along with damage repair. These activities promote and accelerate tissue recovery and functional rehabilitation.

To better describe the aforementioned mechanism, we devised the following virtual experiment, called the "cobweb experiment." This mechanism likens a resting spider's reaction to a damaged cobweb. When the cobweb is touched with a tiny straw, the spider responds by rushing out of the nest toward the "stimulus" in search of its prey. When the spider notices the absence of a prey at the stimulus site but a small mosquito stuck on the web nearby, it will not hesitate to crawl over and devour the mosquito and also mend the proximal damage to the cobweb. With the mosquito consumed, the cobweb is complete again.

Similarly, connective tissue is a continuous network surrounding the organs and tissues throughout the body and similar to the cobweb in structure and function. We hypothesize that the immune response to FSN stimulation of the subcutaneous tissue is similar to the spider's response to a stimulus by a tiny straw. In the experiment, the cobweb acts as the connective tissue, the damaged hole acts as the lesion, the mosquito acts as an external invasion or source of disease, the spider acts as the immune system, and the straw acts as the acupuncture needle.

\section{BASIS OF THE HYPOTHESIS}

The hypothesis of FSN-caused stimulation of the immunological mechanism, the cobweb mechanism, is based on the existing knowledge of histology and physiology, with the theoretical basis as described in the following points.

\section{FSN acupuncture exerts its therapeutic effect by leveraging the body's self-healing mechanism \\ The human body can naturally repair and heal itself, which}

underlies all therapeutic interventions. This view has been widely recognized and accepted by the medical community. Furthermore, all structures have a function, and similarly, all functions have an underlying structure [5]. With regard to the use of acupuncture, connective tissue is the appropriate carrier and structural basis for the human body's self-healing mechanism [1].

FSN does not involve chemotherapeutic or surgical interventions and only mechanical stimulation (intentional minimal invasion). Therefore, the therapeutic effect of FSN can only be achieved by harnessing the body's self-healing and self-recovery abilities. In addition, during FSN treatment, the needles which are inserted into the body's subcutaneous layer lie between the muscles and skin, implying that the only tissue the needles stimulate is the subcutaneous tissue. Therefore, FSN intervention on the subcutaneous tissue stimulates and promotes the body's self-healing mechanism.

\section{Tissue repair is a critical function of the immune system}

The body's immune function consists of 3 aspects: immune defense, immune surveillance, and immune homeostasis. Immune defense refers to body's ability to prevent and remove pathogens such as bacteria, viruses, and fungi. Immune surveillance is body's ability to detect and remove foreign substances, such as tumor cells. Immune homeostasis is maintained through immune tolerance and immunoregulation, that is distinguishing between "self" and "non-self" [6].

Studies have shown that the immune system activity is also crucial to tissue development, self-stability, and damage repair $[7,8]$. Since Metchnikoff's discovery in the late $19^{\text {th }}$ century of the role of macrophages in tissue repair, the involvement of immune cells in tissue repair has been recognized. Recent advances highlight novel mechanisms through which the immune system regulates tissue regeneration [8].

\section{Subcutaneous loose connective tissue is a part of the immune system}

Because the FSN needle primarily affects the subcutaneous loose connective tissue, its therapeutic effect must be closely associated with the histological characteristics of this layer. The structure and function of this tissue likely play a critical role in the effectiveness of FSN treatment. Loose connective tissue consists of randomly arranged fibers and a selection of scattered cells bathed in tissue fluid. This tissue primarily contains 7 types of cells; fibroblasts, macrophages, mast cells, adipocytes, and mesenchymal stem cells are resident cells involved in tissue repair, regeneration, and immunity whereas leukocytes and plasma cells are transient cells (immune cells) involved in effecting immunity [9]. These immune and regenerative cells are activated in response to nociceptive 
stimulation, trauma, and foreign substance invasion. They are activated for immunoreaction, cell differentiation and regeneration, mounting immune response and regulation, and tissue regeneration and repair (Table 1). Therefore, we believe that loose connective tissue contributes to the regeneration and storage of immune cells in the body.

The fibers (including collagenous, elastic, and reticular fibers) and matrix make the interstitium of the connective tissue. The interstitium connects with every organ, from the outside to the inside, from the skin to the internal organs, and it provides a vital nutrient channel and the first line of immune defense [10]. In as early as 2002, Langevin et al. [11] discovered that the connective tissue fibers and matrix are the source and conducting pathways of the signals generated by the acupuncture needle. Benias et al. [10] discovered that the interstitium is full of fluid (matrix), which has conductive properties, and they confirmed that it acts as not only the pathway for the dissemination of cancer cells but also an important channel for the transmission of immune substances within connective tissue, which is of great importance to the immune process. This finding provides a new and powerful theoretical basis for the immune mechanism of FSN acupuncture.

\section{Neuropeptides and neurotransmitters participate in mounting an immune response}

Mechanical intervention and intentional minimal invasion to the subcutaneous tissue, including the nerve endings, are the basis for the therapeutic effect of FSN acupuncture. Studies have shown that nerve endings in the skin and subcutaneous tissue release neuropeptides into the surrounding tissue after stimulation, which can induce local responses such as papulation and erythema [5]. Furthermore, neuropeptides can aggregate "innate immunocytes," neutrophils, and macrophages and participate in the body's immune response with the help of chemokines [5]. The neurotransmitters are secreted by damaged or stimulated sensory nerve endings, modulate pain, and play an important role in immunomodulation; autonomic neurotransmitters such as vasoactive intestinal peptides (VIPs) not only exert strong anti-inflammatory effects, but also participate in immunomodulation by inhibiting the proliferation and migration of T lymphocytes [12].

\section{IMPLICATIONS OF THE HYPOTHESIS}

The hypothesized immunological basis for the therapeutic effect of FSN acupuncture (the cobweb mechanism) may have the following implications for clinical application.

\section{Selection of needlle insertion points}

Given the cobweb-like characteristics of loose connective tissue, FSN acupuncture intervention in any part of the body may induce the body's immune-mediated response to varying extent, which may exert strong or weak therapeutic effects. According to the hypothesized cobweb model, the healthy and complete part of the web should conduct signals faster and more accurately than the damaged part of the web. Therefore, the needle insertion point should be in the nondamaged region but proximal to the damaged region to gain greater efficacy in triggering the local immune response and promoting repair and regeneration. Needle insertion without considering traditional acupuncture points is consistent with the "Pan-acupoint phenomenon" and "Pan-acupoint theory" proposed recently, which state that acupuncture points are not fixed, can exist anywhere on the human body, and certain points may be more effective than others in treating the corresponding organs or conditions [13].

Table 1. Loose connective tissue composition

\begin{tabular}{|c|c|}
\hline Composition & Function \\
\hline \multicolumn{2}{|l|}{ Cells } \\
\hline Macrophages & $\begin{array}{l}\text { Ingest and remove foreign pathogenic substances, cell residues; promote tissue regeneration; secrete } \\
\text { cytokines and contribute to the immune response. }\end{array}$ \\
\hline Mast cells & Participate in allergic response and secrete histamine and cytokines, stimulating inflammatory responses. \\
\hline Plasma cells & $\begin{array}{l}\text { Originate from activated B lymphocytes; produce antibodies and cytokines; participate in humoral immune } \\
\text { response. }\end{array}$ \\
\hline Leukocytes & $\begin{array}{l}\text { Ingest or degrade pathogens and cellular debris; some can also secrete interleukins, interferons and tumor } \\
\text { necrosis factors; participate in inflammation and immunomodulation. }\end{array}$ \\
\hline Fibroblasts & Produce collagenous fibres. \\
\hline Mesenchymal stem cells & $\begin{array}{l}\text { Mainly presented in subcutaneous connective tissue; participate in immunomodulation when activated; } \\
\text { participate in tissue anti-inflammatory, tissue repair and regeneration processes. }\end{array}$ \\
\hline Adipocytes & Store fat and participate in tissue regeneration. \\
\hline \multicolumn{2}{|l|}{ Interstitium } \\
\hline Fibres & Include collagenous, elastic and reticular fibres. \\
\hline Matrix & Contains proteoglycan, glycoprotein and interstitial fluid. \\
\hline
\end{tabular}


In addition, we believe that the intermuscular borders, where the connective tissue conjunctions occur, may collect immune information similar to a cobweb. Stimulating those locations would lead to stronger responses and more efficient conduction of signals because these sites have a high density of connective tissues and are more densely packed with cells, fibers, and matrix.

\section{Expanding the scope of the FSN indications}

The immunological hypothesis for the therapeutic effect of FSN acupuncture indicates that it would likely be effective in disorders other than musculoskeletal disorders. The primary stimulation site for FSN acupuncture is the subcutaneous loose connective tissue. Loose connective tissue forms an anatomic network throughout the body and serves as a bodywide signalling network for mechanical and physical signals [14]. The stimulation of any part of the body will potentially affect the whole body. As proposed above, FSN may enhance the recruitment of immune cells through chemotaxis. FSN stimulation may rapidly generate immune signals which are transmitted by the connective tissue network to the damaged tissues or organs to recruit immune cells and promote recovery processes. This can be likened to the spider when it receives the stimulus from straw intrusion on its web and reacts by rushing out and devouring the mosquito and repairing the damaged web.

Furthermore, according to the current understanding about FSN acupuncture, infectious disease is defined as an absolute contraindication for FSN [1]. However, if the cobweb theory is approbated, FSN acupuncture should not only affect the repair and regeneration mechanism but also yield antiinflammatory effects that could help in both pathogenic and non-pathogenic inflammation. Clinical reports have shown that FSN acupuncture is effective in conditions associated with non-pathogenic tissue inflammation such as adhesive capsulitis, lateral epicondylitis, and arthritis [15]. Although the efficacy of FSN acupuncture on pathogenic inflammation remains unreported, clinical studies exist on the therapeutic efficacy of traditional acupuncture against pathogenic inflammation in diseases such as dysentery and malaria [16-18]. Experimental animal studies have also shown a positive effect of electroacupuncture in the treatment of sepsis [19]. We hypothesize that traditional acupuncture and electroacupuncture effect their anti-infection activity by stimulating the connective tissue. Because the cobweb-like connective tissue is a continuous network in the human body, extending from the subcutaneous tissue to muscles, bones, and internal organs, traditional and electroacupuncture inevitably stimulate the connective tissue. We believe that rigorous clinical and experimental research on this subject are required to establish the potential contribution of FSN acupuncture toward the promotion of anti-inflammatory effects.

\section{The significance of applying appropriate stimulus}

With reference to our spider's web analogy, a weak stimulus may not be adequate to alert the spider. The greater efficacy of FSN than traditional acupuncture could be attributed to greater stimulation rendered by FSN [20]. The FSN needle is approximately 3- to 5-times thicker in diameter than the commonly used acupuncture needle and yields stronger stimulation. During FSN treatment, active resistive exercise of tightened muscles is often prescribed, which is termed the "reperfusion approach." The reperfusion movement while the needle is in the subcutaneous tissue is an adjunct that increases the magnitude of stimulation.

However, an excessively strong stimulation, such as excessive reperfusion, may cause tissue damage, which may not only diminish the positive effects but also aggravate the ailments. With regard to the cobweb analogy, excessive impact of the straw may further damage the web and make repair difficult. Therefore, the magnitude of stimulation from FSN treatment should be considered in clinical practice, with the optimal stimulation decided according to the patient condition and the ailments.

\section{DISCUSSION}

FSN acupuncture is a novel technique based on human physiology, anatomy, and orthodox medicine, which alters the method of selecting needle insertions and the depth of needle insertion compared to traditional acupuncture. FSN acupuncture does not consider traditional acupuncture points and only stimulates the subcutaneous connective tissue to yield its therapeutic effect. Primarily, the tightened muscle theory guides the clinical practice of acupuncture, which proposes that an abnormally high muscular tension reduces the blood supply to the tissues or organs and causes pain and disorders. FSN works by stimulating and pulling the connective tissue to ease the muscular tension and improve blood circulation [1]. While the tightened muscle theory can explain several clinical effects and help in effectively resolving several clinical problems, it has limitations.

The cobweb mechanism theory implies that FSN achieves its therapeutic effect by stimulating the subcutaneous connective tissue, which in turn engages the immune system. Given that the only intervention in FSN is needle manipulation, we hypothesize that FSN achieves its therapeutic effect by engaging self-repair and self-healing mechanisms. Because self-repair and self-healing are also immune functions, this study suggests that FSN's stimulation of the 
subcutaneous connective tissue activates the immune system, which exerts the therapeutic effects.

FSN engages the immune system by merely stimulating the subcutaneous layer. The primary functional basis of the hypothesized cobweb mechanism is the unique histological and physiological characteristics of the layer of loose connective tissue that all the connective tissue cells participate in tissue regeneration and immune activity. The cells likely contribute to the processes after activation by FSN acupuncture.

Connective tissues constitute a network throughout the body, including intermuscular and subcutaneous tissue, and are continuous with more specialized connective tissues such as perimysium, periosteum, pleura, and peritoneum [21]. The analogy with the spider's response to an external stimulus on its web is based on the fact that connective tissue and the cobweb are remarkably similar in structure.

Apart from connective tissue, other tissues stimulated by FSN manipulation are the subcutaneous nerve endings. Evidence suggests that mechanical stimulation of nerve endings can lead to a local inflammatory response and the aggregation of local immune cells that participate in the anti-inflammatory mechanism through the release of neuropeptides and neurotransmitters [5,12]. This constitutes another physiological basis for our immunological hypothesis of FSN's therapeutic effect.

Regarding the selection of the needle insertion site, given that the intermuscular borders are considered as the most effective insertion sites, we hypothesize that an insertion site as proximal as possible to the lesion will yield highest efficacy. The concept of an intermuscular border, named Fen-Rou in ancient Chinese, has been frequently mentioned in ancient Chinese medical literature; for instance, intermuscular borders have been described as the meeting points of the stream and the valley (Suwen, Discussion of acupoints) [22]. Several scholars have described these as the "travelling places of meridians"; however, some others emphasize these as descriptions of acupoints in ancient times [23]. This view is supported by several biological studies on acupuncture, which have found that classic acupoints and meridians are typically located between muscles or between muscles and bones, and studies indicate that the ancient maps of acupuncture points and meridians might essentially be a guide to inserting the needle into connective tissue [22].

\section{CONCLUSIONS}

This work proposes that FSN stimulates the immune system to activate the self-healing mechanisms of the human body to treat a broad range of disorders. To aid in the comprehension of this concept, a cobweb analogy has been used to illustrate the therapeutic roles of FSN acupuncture. Further experimental study to demonstrate the underlying molecular mechanism of FSN treatment is required.

\section{FUNDING}

This research did not receive any specific grant from funding agencies, either public or commercial.

\section{ACKNOWLEDGEMENTS}

Our sincerely thanks to Dr. Antony Warford, Emeritus Fellow, Dept of Life Sciences at the University of Westminster for providing valuable feedback on this paper.

\section{CONFLICT OF INTEREST}

The authors declare no conflict of interest.

\section{ORCID}

Wenbo Xu, Jidong $\mathrm{Wu}$, Ping Xu,

https://orcid.org/0000-0002-4715-6745 https://orcid.org/0000-0002-1000-1264 https://orcid.org/0000-0003-0609-0273

\section{REFERENCES}

1. Fu Z. [The Foundation of Fu's Subcutaneous Needling]. Beijing: People's Medical Publishing House, 2016. Chinese.

2. Travell JG, Simons DG. Myofascial Pain and Dysfunction: The Trigger Point Manual. Baltimore: Williams and Wilkins, 1983.

3. Fu Z, Wu J, Mearns AB, Wu D. Under the Skin: A Manual of Fu's Subcutaneous Needling (FSN) Acupuncture. Beijing: People's Medical Publishing House, 2020.

4. Xu W, Wu J. [Theoretic study on the immuno-modifying effect of Fu's subcutaneous needling acupuncture]. J Chin Med Acupunct 2017;24:1-3. Chinese.

5. Zhu B. [Systematic Science of Acupuncture and Moxibustion]. Beijing: People's Medical Publishing House, 2015. Chinese.

6. Cao X. [Medical Immunology], 6th ed. Beijing: People's Medical Publishing House, 2013. Chinese.

7. Murray PJ, Wynn TA. Protective and pathogenic functions of macrophage subsets. Nat Rev Immunol 2011;11:723-37.

8. Aurora AB, Olson EN. Immune modulation of stem cells and regeneration. Cell Stem Cell 2014;15:14-25.

9. Rad A. Loose connective tissue. Available at: https://www. kenhub.com/en/library/anatomy/loose-connective-tissue [Date accessed: October 29, 2020]

10. Benias PC, Wells RG, Sackey-Aboagye B, Klavan H, Reidy J, Buonocore D, et al. Structure and distribution of an unrecognized interstitium in human tissues. Sci Rep 2018;8:4947. 
11. Langevin HM, Churchill DL, Wu J, Badger GJ, Yandow JA, Fox JR, et al. Evidence of connective tissue involvement in acupuncture. FASEB J 2002;16:872-4.

12. Ackermann PW. Neuronal regulation of tendon homoeostasis. Int J Exp Pathol 2013;94:271-86.

13. Li Y, Gong C. [Dialogue on the development of acupuncture and moxibustion in the United States]. Guid J Tradit Chin Med Pharm 2019;17:1-6. Chinese.

14. Langevin HM. Connective tissue: a body-wide signaling network? Med Hypotheses 2006;66:1074-7.

15. Kou Z, Zhao M, Zhang X. [Application and analysis of floating needle therapy in neck,shoulder,waist and leg pain]. J Rheum Arthritis 2020;(3):77-80. Chinese.

16. Qiu M, Sheng C, Li N, Wang JM, Wang JS, Wu D, et al., eds. A Study of Acupuncture Treatment for Acute Bacillary Dysentery. National Symposium of Acupuncture, Moxibustion, and Acupuncture Anaesthesia, June 1-5, 1979, Beijing, China. Beijing: People's Medical Publishing House, 1980.

17. $\mathrm{Lu} \mathrm{Z}$. [Research on immune mechanism of acupuncture in the treatment of acute bacillary dysentery]. J Med Res 1984;10:16-7. Chinese.

18. Sun G. [Acupuncture and Moxibustion]. Beijing: People's Medical Publishing House, 2000. Chinese.

19. Torres-Rosas R, Yehia G, Peña G, Mishra P, del Rocio Thompson-Bonilla M, Moreno-Eutimio MA, et al. Dopamine mediates vagal modulation of the immune system by electroacupuncture. Nat Med 2014;20:291-5.

20. Fu Z, Huang Y. [Comparing the efficacies of FSN and traditional acupuncture on subacromial bursitis]. J Tradit Chin Med Univ Hunan 1999;19:60-1. Chinese.

21. Langevin HM, Churchill DL, Cipolla MJ. Mechanical signaling through connective tissue: a mechanism for the therapeutic effect of acupuncture. FASEB J 2001;15:2275-82.

22. He Q. [The Medical Masterpieces of the Past Generations]. Haikou: Hainan International Press and Publication Centre, 1996. Chinese.

23. Zhang SJ. [Early morphological concepts of acupoint]. Zhongguo Zhen Jiu 2011;31:1127-30. Chinese. 